\title{
Cancer prevention: when knowledge of cancer prevention is not enough
}

\author{
Xue Qin Yu, PhD MPH ${ }^{1,2^{*}} ;$ Peter Baade, $\mathrm{PhD}^{3,4,5}$ \\ ${ }^{1}$ Cancer Research Division, Cancer Council NSW, Sydney, Australia \\ ${ }^{2}$ Sydney School of Public Health, Faculty of Medicine and Health, University of Sydney, \\ Sydney, Australia. \\ ${ }^{3}$ Cancer Research Centre, Cancer Council Queensland, Brisbane, Australia \\ ${ }^{4}$ Menzies Health Institute Queensland, Griffith University, Gold Coast Campus, Southport, \\ Australia \\ ${ }^{5}$ School of Mathematical Sciences, Queensland University of Technology, Brisbane, \\ Australia
}

* Corresponding author and address:

Dr Xue Qin Yu,

Cancer Council NSW

P O Box 572

Kings Cross, NSW 1340 Australia

Phone: 61-2-9334 1851

Fax: $61-2-83023550$

Email: xueqiny@nswcc.org.au

\section{Conflicts of interest}

None declared.

\section{Funding}

This editorial has not received any funding, and the first author is employed by Cancer Council NSW, Australia and the second author by Cancer Council Queensland, Australia. 
Cancer places a significant economic burden on the health care system in China, with over 3.8 million new cases diagnosed and nearly 2.3 million cancer deaths occurring in $2014 .^{1}$ This burden is expected to increase further in the near future due to the aging population and the increasing trend in urbanization in China. ${ }^{2} \mathrm{~A}$ recent study indicates that almost half of all cancer deaths (52\% for men and 35\% for women) in China in 2013 were attributable to potentially modifiable risk factors such as smoking, infections and alcohol consumption. ${ }^{3}$ Effective public health interventions to eliminate or reduce an individual's exposure to these risk factors will therefore be critical to reducing the future cancer burden in China.

It is within this context that we turn to the current study by Li et al., ${ }^{4}$ who examined the association between levels of cancer prevention knowledge and incidence of cancer among a large cohort surveyed across four provinces in China. An important strength of the study is the prospective study design involving a large cohort of cancer screening attendees $(164,341)$ with a very high follow-up rate $(>90 \%)$. The study found that groups with higher levels of cancer prevention knowledge had a lower risk of cancer diagnosis after a median of 3.3 years follow-up. The overall diagnosis rate in the group with the highest knowledge scores $(1.5 \%)$ was nearly half that for those with the lowest knowledge scores $(2.9 \%)$. After adjusting for potential confounders (age, sex, individual-level socioeconomic indicators of education and income, and family history of cancer), those in the groups with higher cancer prevention knowledge at baseline still had a significantly lower risk of being diagnosed with cancer (adjusted hazard ratio ranging from 0.78 to $0.67 ; p<0.0001$ ) than those in the lowest knowledge group.

These results suggest that increasing the population's knowledge and awareness of cancer prevention will assist efforts to reduce the high cancer burden in China. However, there are some important points to note when considering the practical implications of these results. 
On a methodological level, due to the typically longer (but usually unknown) lag time between risky behavior (exposure) and cancer diagnosis, ${ }^{5}$ the limited length of follow-up in the study by Li et al. ${ }^{4}$ suggests that it is unlikely that current levels of cancer prevention knowledge would have directly affected the cancer incidence reported. Rather it may be that the measured current knowledge is generally consistent with their general awareness over previous years, and this previous knowledge is what is associated with the observed risk of cancer diagnosis. When designing interventions based on increasing knowledge, some caution may be needed about the probably lengthy time interval before the impact of the intervention would be expected.

Given the high general awareness about the link between tobacco smoking and lung cancer, it was initially counterintuitive that there was no significant association found between knowledge levels and incidence of lung cancer (Supporting Information Table 2). Notably, the authors did not give a specific explanation for this result. In our opinion, the result may reflect the particularly long time interval between exposure of tobacco smoking and lung cancer diagnosis, as it has been suggested that lung cancer incidence trends reflect the smoking trends of 20-30 years earlier. ${ }^{6}$ It may also be that knowledge of the dangers of smoking is not enough to overcome the strong cultural norm of smoking among Chinese men, reflected by that in many regions, especially rural China, cigarettes are still considered to be a common gift to friends and relatives. ${ }^{7}$ Finally, there is a low prevalence of quitting behavior among current smokers, ${ }^{8}$ possibly caused by the higher levels of tobacco dependence among Chinese male smokers than smokers from other countries, ${ }^{9}$ together with the lack of established tobacco cessation programs in China. ${ }^{7}$ Taken together, these reasons may reduce the opportunity and motivation of smokers in China to quit irrespective of their knowledge levels.

In this study, only participants assessed as high-risk at the initial screening were referred for clinical examination (secondary screening), even though cases were ascertained by linkage 
to population-based cancer registries. One of the typical outcomes of any cancer screening program is observing a greater diagnosis rate of asymptomatic, localized cancers. Without any information about the stage of cancer at diagnosis, it is not possible to determine whether there may be a screening effect present in these results. However, if there was, it would require high risk screening attendees to also be more likely to have high cancer prevention knowledge, so this explanation is unlikely. Longer follow-up intervals, and stratification of results by high- and low-risk groups, would undoubtedly provide a clearer picture.

The reported difference in the relationship between cancer prevention knowledge and cancer diagnosis for males and females provides further evidence that there are complexities in this relationship which must be further explored. While an inverse association between cancer prevention knowledge and cancer diagnosis was observed among female respondents, the opposite was true for males. This may be due to males in China tending to have a substantially higher prevalence of risk factor exposure, such as smoking and alcohol consumption and exposure to occupational hazards, ${ }^{3,10}$ but it is difficult to be sure of how this relates to cancer prevention knowledge and what it means for its use as a mechanism for reducing the cancer burden. This highlights the critical and complex link between knowledge and behavior. While increasing knowledge has the potential to motivate behavior, any intervention based on an increase in knowledge only, without providing the means or opportunity to also change behavior, will have limited utility. ${ }^{11}$

$\mathrm{Li}$ et al. also found that the association between the level of cancer knowledge and the risk of developing cancer differed by education and income levels, as well as family history of cancer. ${ }^{4}$ Interestingly, the inverse effect of level of cancer prevention knowledge on risk of cancer diagnosis was evident in the subgroups that would typically be associated with lower awareness and knowledge - those with poor education, low income and no family history of cancer. ${ }^{12}$ This unusual association may explain the results, as the participants with high 
knowledge in these disadvantaged groups would provide a much greater contrast to the rest of their cohort than those with high knowledge in groups with higher socioeconomic advantage. The distribution of knowledge levels by income status supports this hypothesis, as $12 \%$ of the lower income group were in the lowest knowledge group and $12 \%$ were in the highest knowledge group, while for the higher income group the equivalent proportions were $4 \%$ and $17 \%$. Even if people with higher socioeconomic status have poor knowledge about cancer prevention, they typically would have the benefits of better general health and wellbeing due to that higher income and/or education, ${ }^{13}$ so the impact of that lack of cancer prevention knowledge would be mitigated. These results should provide further motivation for policy makers to develop programs to increase knowledge about cancer prevention, as the impact of increased knowledge would be greatest for those who need it most.

We note that the association between cancer prevention knowledge levels at baseline and subsequent risk of cancer diagnosis varied by cancer type, with prominent associations observed for stomach cancer and (among women) breast cancer. The authors have explained this using the health belief model, ${ }^{14}$ which attempts to predict why people will take action to prevent, screen or control health conditions. This model would suggest that to reduce their risk of developing cancer, participants with higher awareness of the risk factors for stomach cancer would then be more motivated to take action to avoid those unhealthy lifestyles, such as tobacco smoking, alcohol consumption, and excessive intake of salted and pickled foods.

The measure of awareness of cancer prevention and risk factors used in this study was developed based on reports by the National Health Commission of China, input from an expert panel, and a previously evaluated questionnaire.$^{15}$ However, given its content validity and reliability in the Chinese population has not yet been assessed, it is possible that the knowledge measure used in this study does not completely reflect the important constructs of cancer prevention knowledge that impact behavior in the Chinese population. Further 
research to validate this measure, and to better understand the factors which influence health behaviors in this population are warranted.

As four of the five most commonly diagnosed cancers in China ${ }^{1}$ (lung, stomach, liver and esophagus) have established risk factors, ${ }^{3}$ limited treatment options ${ }^{16,17}$ and high case fatality, ${ }^{18}$ reducing the burden of these cancers in the long term may be most successfully achieved through primary prevention strategies and earlier detection. While knowledge itself is not sufficient for change, both primary prevention and early detection activities rely on a foundation of knowledge in the population. Thus, increasing public awareness of cancer risk by delivering tailored health and lifestyle advice, as well as providing the means or opportunity to change risky behaviors, will be crucial to reducing the burden of cancer. All levels of governments in China have a key role to play in raising awareness of common risk of cancers and their early symptoms as well as preventive measures to reduce cancer risk. This paper by Li and colleagues ${ }^{4}$ should provide further motivation to develop strategies to increase knowledge. However, since increasing knowledge alone may not be enough for generating behavioral change of sufficient magnitude to have a real impact on cancer risk, more cancer prevention research thus is needed ${ }^{19}$ to identify and understand the barriers that restrict the link between knowledge and behavioral change.

However, action can be taken now. There already exist some proven effective populationbased policies that can be implemented to change behavior. Examples include the National Smoke-free Regulation, which bans smoking in public places including schools, restaurants and work places and has been adopted recently. ${ }^{20}$ However, adoption and implementation of this regulation is likely to be ineffective unless there are incentives to do so. Evidence from 125 Chinese cities where subnational smoke-free laws are adopted indicated that public enforcement for the smoke ban has not been enacted effectively, with the current adoption of the regulation accompanied by inadequate supervision measures and punishments for 
violations, leading to calls for increased enforcement of the smoke-free regulations in China. ${ }^{20}$

This study demonstrated that the level of knowledge for some cancer related issues was fortunately already very high, such as $90 \%$ of participants knowing that a lump or pain might be early symptoms of breast cancer. However, in other areas the level of knowledge was much lower, with, for example, only $40-45 \%$ of participants knowing that infection was an important risk factor for liver cancer and a vaccine for hepatitis was available as a preventive measure. Given that liver cancer is one of the major cancers in China, with very high mortality rates, increasing the level of public awareness of these cancer risks and their preventive measures should remain a priority, particularly in geographical areas or population subgroups where the burden is higher.

It has long been recognized that knowledge of cancer could influence an individual's lifestyle and health behaviors, ${ }^{21}$ and it is hoped that this impressive study by Li and colleagues ${ }^{4}$ will motivate action by policy makers and medical professionals to use cancer knowledge as a tool in the fight against cancer. However, it is important that not only are levels of cancer prevention knowledge increased in the community, but also that key barriers to subsequent behavioral change be identified and removed if the massive cancer burden in China is to be addressed. 


\section{References}

1. Chen W, Sun K, Zheng R, et al. Cancer incidence and mortality in China, 2014. Chin J Cancer Res. 2018;30: 1-12.

2. Tsoi KK, Hirai HW, Chan FC, Griffiths S, Sung JJ. Cancer burden with ageing population in urban regions in China: projection on cancer registry data from World Health Organization. Br Med Bull. 2017;121: 83-94.

3. Islami $F$, Chen W, Yu XQ, et al. Cancer deaths and cases attributable to lifestyle factors and infections in China, 2013. Ann Oncol. 2017;28: 2567-2574.

4. Li H, Zeng H, Zheng R, et al. Association of Cancer Awareness Levels with Risk of Cancer in Rural China: A population-based cohort study. Cancer. 2020;126: 000-000.

5. Giovannucci E, Rimm EB, Stampfer MJ, et al. A prospective study of cigarette smoking and risk of colorectal adenoma and colorectal cancer in U.S. men. J Natl Cancer Inst. 1994;86: 183-191.

6. Youlden DR, Cramb SM, Baade PD. The International Epidemiology of Lung Cancer: geographical distribution and secular trends. J Thorac Oncol. 2008;3: 819-831.

7. Parascandola M, Xiao L. Tobacco and the lung cancer epidemic in China. Transl Lung Cancer Res. 2019;8: S21-S30.

8. China Center for Disease Control and Prevention. China Adult Tobacco Survey Report. Beijing, China, 2015.

9. ITC Project and Tobacco Control Office CC. ITC China Project Report. Findings from the Wave 1 to 5 Surveys (2006-2015). In: University of Waterloo W, Ontario, Canada, and Tobacco Control Office, Chinese Center for Disease Control and Prevention, Beijing, China, editor, 2017.

10. Li J, Yin P, Wang $\mathrm{H}$, et al. The disease burden attributable to 18 occupational risks in China: an analysis for the global burden of disease study 2017. Environ Health. 2020;19: 21.

11. Kelly MP, Barker M. Why is changing health-related behaviour so difficult? Public Health. 2016;136: 109-116.

12. Robb K, Stubbings $S$, Ramirez A, et al. Public awareness of cancer in Britain: a population-based survey of adults. Br J Cancer. 2009;101 Suppl 2: S18-23.

13. Adler NE, Newman K. Socioeconomic disparities in health: pathways and policies. Health Aff (Millwood). 2002;21: 60-76.

14. Rosenstock IM, Strecher VJ, Becker MH. Social learning theory and the Health Belief Model. Health Educ Q. 1988;15: 175-183.

15. Stubbings S, Robb K, Waller J, et al. Development of a measurement tool to assess public awareness of cancer. Br J Cancer. 2009;101 Suppl 2: S13-17.

16. Zeng $\mathrm{H}$, Chen $\mathrm{W}$, Zheng $\mathrm{R}$, et al. Changing cancer survival in China during 2003-15: a pooled analysis of 17 population-based cancer registries. Lancet Glob Health. 2018;6: e555-e567.

17. Zeng H, Zheng R, Guo Y, et al. Cancer survival in China, 2003-2005: a population-based study. Int J Cancer. 2015;136: 1921-1930.

18. Chen W, Zheng R, Baade PD, et al. Cancer statistics in China, 2015. CA Cancer J Clin. 2016;66: 115-132.

19. Yu S, Yang CS, Li J, et al. Cancer Prevention Research in China. Cancer Prev Res (Phila). 2015;8: 662-674.

20. Lin H, Chang C, Liu Z, Zheng Y. Subnational smoke-free laws in China. Tob Induc Dis. 2019;17: 78.

21. Paul C, Tzelepis F, Walsh RA, Girgis A, King L, McKenzie J. Has the investment in public cancer education delivered observable changes in knowledge over the past 10 years? Cancer. 2003;97: 2931-2939. 